\title{
Verification of thermoelectric magnetohydrodynamic flow effects on dendritic tip kinetics by in-situ observations
}

Rijie Zhao ${ }^{\mathrm{a}}$, Jianrong Gao ${ }^{\mathrm{a},{ }^{*}, \text { Andrew Kao }}{ }^{\mathrm{b}}$, Koulis Pericleous ${ }^{\mathrm{b}}$

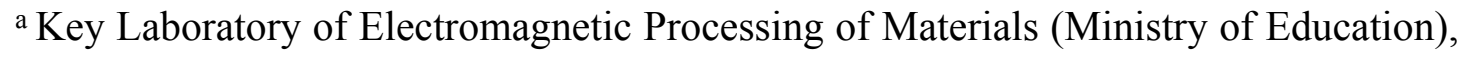
Northeastern University, Shenyang 110819, China

b Centre for Numerical Modelling and Process Analysis, University of Greenwich, London SE10 9LS, United Kingdom

\begin{abstract}
Magnetohydrodynamic flows can be driven by Lorentz forces acting on thermoelectric currents. Their effects on tip velocities of Pd dendrites solidifying from an undercooled melt under magnetic field intensities of up to $6 \mathrm{~T}$ were investigated by in-situ observations using a high-speed camera. At low undercoolings, the tip velocities are depressed by magnetic fields of low and high intensities, but recover under an intermediate magnetic field intensity. At high undercoolings, the tip velocities are also depressed, but their recovery is shifted to a higher magnetic field intensity. These observations on $\mathrm{Pd}$ dendrites support and extend the findings obtained in previous studies on $\mathrm{Ni}$ and Fe dendrites, and fully verify the convection effects on dendritic growth due to three thermoelectric magnetohydrodynamic flow patterns as predicted in recent numerical simulations.
\end{abstract}

Keywords: Dendrites; magnetic fields; tip velocities; thermoelectric magnetohydrodynamic flows; in-situ observations

* Corresponding author. E-mail address: jgao@mail.neu.edu.cn (J. Gao) 


\section{Introduction}

Thermoelectric magnetohydrodynamics (TEMHD) in an electrically conducting liquid was first drawn to attention by Schercliff $[1,2]$. It can occur in solidification of liquid metals under static magnetic fields, where thermoelectric currents are the result of temperature differences and discontinuity in electron affinity crossing the phase boundary of a growing crystal [3, 4]. Like buoyancy-driven natural flows [5], TEMHD flows driven by the Lorentz forces can alter heat and mass transfer ahead of the crystal/liquid interface and modify the morphology and segregation patterns of the crystal. The influence of TEMHD flows on crystal growth was observed in directional solidification of metallic alloys, where it aroused much research interest [3, 6-12]. The microscopic mechanisms underlying the influence were revealed in numerical simulations [13-16]. Recent simulations [15] highlighted the formation of three kinds of TEMHD flow patterns in undercooled dendritic growth. As illustrated in Fig. 1, a transverse flow is most dominant at low magnetic field intensities, bringing hot liquid to the top of a primary tip and leading to a depression of the tip velocity. As the magnetic field intensity is increased to a moderate level, a rotational flow becomes dominant. It can counterbalance the effect of the transverse flow allowing the recovery of the depressed tip velocity. Further increase in magnetic field intensities leads to damping of the transverse flow and confinement of the rotational flow in a thin liquid layer. Consequently, the dendritic tip is coarsened and the tip velocity is reduced. Gao et al. $[17,18]$ investigated the effects of the TEMHD flows on tip velocities of undercooled $\mathrm{Fe}$ and $\mathrm{Ni}$ dendrites using a high speed-camera. Their 
observations of a depression and a recovery of dendrite tip velocities verified the effects of the transverse and the rotational flow, respectively. However, the coarsening effect predicted for high magnetic field intensities [15] is not observed due to experimental constraints. In the work presented here, tip velocities of undercooled Pd dendrites were measured. The measured data provided evidence for the first time for the effects of all three kinds of TEMHD flow patterns in a single material and fully verified the predictions of the recent numerical simulations [15].

\section{Material and Method}

A sphere-like sample of nominally pure Pd (99.9\% purity) with a mass of $1 \mathrm{~g}$ was used for measurements of dendritic tip velocities with or without static magnetic fields. The experimental set-up is shown in Fig. 2. The sample was inductively heated, melted and overheated under protection of an argon atmosphere. The liquid sample was undercooled using the glass-fluxing technique [19]. A small amount of soda lime glass pieces was placed between the sample and a ceramic-made sample holder. The glass pieces were fused by the heated sample and wetted the sample surface. The surface temperature of the sample was measured using a single-color pyrometer at a sampling rate of $100 \mathrm{~Hz}$ and was calibrated using a method described elsewhere [19]. Rapid solidification of the undercooled sample brought about strong recalescence due to rapid release of latent heat $[17,18]$. The recalescence process of the sample surface was in-situ monitored using a high-speed camera with a maximum frame rate of $100,000 \mathrm{fps}$ and a pixel size of $100 \mu \mathrm{m} \times 100 \mu \mathrm{m}$. Static magnetic fields of intensities 
ranging from $\mathrm{B}=0 \mathrm{~T}$ to $\mathrm{B}=6 \mathrm{~T}$ were generated using a commercial superconducting magnet with a bore of $300 \mathrm{~mm}$ in diameter. In the glass-fluxing experiment, a given magnetic field intensity was excited at the position of the sample and held constant through melting and solidification of the sample. The sample was melted and solidified more than 20 times under each magnetic field intensity to obtain a wide spectrum of undercooling. The recorded digital video images were analyzed using a Matlab-based computer program to determine tip velocities, $\mathrm{V}$, of undercooled dendrites at different undercoolings, $\Delta \mathrm{T}[17,18]$. The principle and details of this method are explained below.

As illustrated in Fig. 3, an undercooled dendrite was nucleated on the bottom side of the sample surface. It grew with an equiaxed morphology towards the top side of the sample surface. This equiaxed morphology could be approximated by a pyramid, of which one half was truncated by the sample surface. Meanwhile, diffusion of released latent heat brought about a thermal front ahead of the growing dendrite. This thermal front moved itself like a traveling spherical wave that was emitted from the nucleation site of the dendrite. Although the thermal front travelled faster than the dendrite grew, the distance between them was small. For example, it was estimated to have a magnitude of $0.1-100 \mu \mathrm{m}$ assuming liquid thermal diffusivity of $10^{-5} \mathrm{~m}^{2} / \mathrm{s}$ and tip velocities of $0.1-100 \mathrm{~m} / \mathrm{s}$. This magnitude of the distance was smaller than or comparable to the pixel width of $100 \mu \mathrm{m}$ of the high-speed camera video images. Thus, the travelling speed of the thermal front was supposed to give a good approximation of the tip velocity of the growing dendrite. Under this assumption, the 
tip velocity of the growing dendrite was determined by computer simulations of the motion of the thermal front in three-dimensional space. The thermal front inside the bulk liquid was not seen and neither the equiaxed dendrite was. However, it had intersections with the sample surface which were discernible. The motion of the intersected thermal front was recorded in the video images with high accuracy. Then, the motion of the thermal front inside the bulk sample could be reconstructed by digital simulations of the video images using the computer software. As a first step of the reconstruction, a few locations on the sample surface were tentatively assigned to be candidate nucleation sites. Then, spherical waves emitting from them were simulated in a sphere that had a size identical to that of the real sample. Where any set of spherical waves provided a good imitation of the observed thermal front loci on the sample surface at the framerate chosen from the video images, a hypothesized nucleation site was accepted as the real one. With the knowledge of the nucleation site, the traveling distance and time of the thermal front inside the bulk sample could be simulated in sequence of the recorded video images. Last, a linear law was fitted to the computed thermal front distance-time relationship. The slope of the linear law defined the traveling speed of the thermal front. As explained above, this traveling speed could be taken as the tip velocity of the undercooled dendrite in the real sample. Uncertainties of the determined tip velocities from different sources are discussed in Section 3.

\section{Results}


Figure 4 illustrates video images of the top surface of the sample recorded during recalescence under different conditions of undercooling and magnetic field intensity. Each image comprised a bright part and a dark part of the sample surface. The two parts corresponded to a recalescing surface area and the undercooled liquid, respectively. A boundary between them was the thermal front. Similar to previous observations on samples of other metals [17-19], the thermal front showed a zigzagged and a relatively smooth morphology at low and high undercoolings, respectively. Such undercooling-dependent morphologies of the thermal front were observed under each magnetic field intensity. As explained in Section 2, a thermal front distance-time relationship was determined by computer simulations of the sequentially recorded video images of each recalescence process. As shown in the right-hand panels of Fig. 4, a linear law gave a reasonable fitting to the relationship. This linear fitting suggested that the tip velocity was constant through recalescence of the bulk sample. The tip velocity determined using this method had errors of different origins. One error was due to the irregular zigzagging of the thermal front. This geometrical irregularity increased difficulties in simulating the thermal front loci using a simple spherical wave approximation and brought about a considerable error of the tip velocity. To minimize this error, the simulation was repeated several times and an averaged slope was taken as the tip velocity. Then the error itself could be defined as the root-mean-square (RMS) error of the slopes derived from the repeated simulations. The RMS error had a dependence on undercooling as the zigzagging of the thermal front became less pronounced with rising undercooling. To quantize this 
dependence, a relative error was defined by dividing the RMS error over the tip velocity. For the tip velocities measured under each magnetic field intensity, their relative errors could be minimized down to $15 \%$ for low undercoolings, e.g. $\Delta \mathrm{T}<50$ $\mathrm{K}$, whereas they could be reduced down to $5 \%$ or below for high undercoolings, e.g. $\Delta \mathrm{T}>100 \mathrm{~K}$. Note that the nucleation sites showed a distinct scatter among different recalescence processes. This scatter was understood because heterogeneous nucleation has a stochastic nature. A triggering of nucleation may avoid this scatter, but was hard to practice in the present experiment due to restricted space inside the vacuum chamber of the experimental set-up. Thus, the scatter of the nucleation sites remained under each magnetic field intensity. This scatter brought about two consequences on the measured data. On one hand, it brought about a scatter of the angle between the tip growth direction and a thermal gradient occurring along the height of the sample [19]. The latter introduced a scatter of the contribution of the thermal gradient to the bulk undercooling [19] and therefore a scatter of the tip velocity at an apparent undercooling measured by the pyrometer. On the other hand, it brought about a scatter of the angle between the tip growth direction and the direction of the magnetic field and therefore a scatter of the tip velocity by affecting competition between different kinds of flow patterns [15]. Assuming a random scatter of the nucleation sites, the resultant scatter of the tip velocity could be filtered by fitting powers laws to the tip velocity-undercooling relationships under each magnetic field intensity. As shown below, this filtering was effective and allowed for determination of different kinds of TEMHD flow effects on dendritic growth. 
Figure 5 shows the tip velocities of Pd dendrites measured under different magnetic field intensities. The data exhibit a two-staged undercooling dependence generally. A slow kinetics is replaced by a rapid one at a critical undercooling of $\Delta \mathrm{T}_{\text {crit }}=110 \mathrm{~K}$. Such a transition is typically observed for dendrites growing from a dilute alloy melt $[20,21]$. Its occurrence in the present sample suggested that impurities dissolved in the melt behave like a solute and induce a transition of tip kinetics upon complete solute trapping. The imposition of the static magnetic fields preserves the two-staged undercooling dependence of the tip velocities and keeps $\Delta \mathrm{T}_{\text {crit }}$ constant within a relative accuracy of $\pm 6 \mathrm{~K}$ of the pyrometric measurements. As explained above, a scatter of the tip velocities measured under similar conditions of undercooling and magnetic field intensity was related to a scatter of the nucleation sites of undercooled dendrites and could be filtered by fitting a series of power laws to the tip velocities. A single power law was fitted to the data measured either at lower $\left(\Delta \mathrm{T}<\Delta \mathrm{T}_{\text {crit. }}\right)$ or at higher $\left(\Delta \mathrm{T}>\Delta \mathrm{T}_{\text {crit. }}\right)$ undercoolings for each magnetic field intensity. The power laws have a general formalism of $\mathrm{V}=a \cdot \Delta \mathrm{T}^{b}$, where $a$ and $b$ are adjustable coefficients. As listed in Table 1, the two coefficients of the power laws are more dependent on undercooling than on the intensity of the magnetic fields. This stronger dependence on undercooling can be understood because the magnetic field intensities of a few Teslas hardly change the Gibbs free energies of solid and liquid metals due to small magnitudes of their magnetic susceptibilities at temperatures close to melting [22]. Root-mean-square deviations of the measured tip velocities relative to the power lawfitted tip velocities are also listed. These deviations give a good measure of the errors 
of the measured tip velocities in the entire undercooling regime. The deviations of the tip velocities measured without a magnetic field are most likely to represent a sum of two kinds of error: the error arising from non-ideal simulations of the irregular morphology of the thermal front and the error due to the scatter of the liquid undercooling. The two kinds of error are comparable at low undercoolings because the first kind has a relative magnitude of $15 \%$. On the other hand, the deviations of the tip velocities measured under the magnetic fields are by $5 \%$ larger or smaller than the deviations of the data measured without a magnetic field. Such small changes of the deviations suggested that the errors due to the scatter of the tip growth direction relative to the magnetic field direction may be smaller than the errors due to other origins. The dependence of the two coefficients of the fitted power laws on the magnetic field intensity provided experimental evidence for the effects of TEMHD flows on tip velocities. It suggested relative changes of the tip velocities typically larger than the root-mean-square deviations of the measured data. As explained below, these relative changes of the tip velocities fully verified for the first time all three kinds of TEMHD flow effects that were predicted by the numerical simulations [15].

The power law-fitted tip velocities under each magnetic field intensity were normalized with respect to those without a magnetic field. The fitted tip velocities are truncated at a limiting undercooling of $\Delta \mathrm{T}=220 \mathrm{~K}$. The reason for this truncation is that the field-dependent variations of the normalized tip velocities above the limiting undercooling become comparable to the root-mean-square deviations of the measured tip velocities (see Table 1). As are plotted in Fig. 6, the normalized tip velocities at 
given undercoolings show significant variations with rising magnetic field intensity. For undercoolings below $\Delta \mathrm{T}=70 \mathrm{~K}$, the normalized tip velocities are depressed by lower magnetic field intensities of $\mathrm{B}=1-3 \mathrm{~T}$ and recover under higher magnetic field intensities of $\mathrm{B}=3-5 \mathrm{~T}$ (see Fig. 6a). Such a depression and a recovery of the tip velocities with rising magnetic field intensity is similar to that observed previously for dendrites of $\mathrm{Ni}$ and $\mathrm{Fe}[17,18]$. It can be attributed to the effects of a transverse flow and a rotational flow, respectively [13-15]. However, the normalized tip velocities of Pd dendrites are again depressed by the highest magnetic field intensity of $\mathrm{B}=6 \mathrm{~T}$. This second depression was not observed in previous studies of $\mathrm{Ni}$ and Fe dendrites $[17,18]$. In terms of the recent numerical simulations [15], it can be attributed to the coarsening effect of a confined rotational flow, which occurs preferably at low tip velocities and high magnetic field intensities. However, the second depression becomes blurred as undercooling is increased to $\Delta \mathrm{T}=70 \mathrm{~K}$ or above. It is very likely to be shifted to magnetic field intensities of higher than $\mathrm{B}=6 \mathrm{~T}$. Consequently, the normalized tip velocities show only the first depression and the subsequent recovery in the undercooling range $70-200 \mathrm{~K}$. At the critical undercooling of $\Delta \mathrm{T}_{\text {crit }}=110 \mathrm{~K}$, the significant depression is caused by higher magnetic field intensities of $\mathrm{B}=3-5 \mathrm{~T}$. Meanwhile, the recovery of the fitted tip velocities is shifted to the highest magnetic field intensity of $\mathrm{B}=6 \mathrm{~T}$. At a slightly higher undercooling of $\Delta \mathrm{T}=120 \mathrm{~K}$, the largest depression is induced by a magnetic field intensity of $\mathrm{B}=4 \mathrm{~T}$ (see Fig. 6b). For undercoolings of $\Delta \mathrm{T}=130-190 \mathrm{~K}$, the depression becomes less and less pronounced with rising undercooling. However, the second depression is seemingly induced by 
the highest magnetic field intensity for undercoolings of $\Delta \mathrm{T}=200-220 \mathrm{~K}$. It becomes comparable to the root-mean-square deviations of the measured data and requires verification with more precise measurements.

\section{Discussion}

The present results showed the significant influence of the magnetic fields on tip velocities of $\mathrm{Pd}$ dendrites for undercoolings up to $\Delta \mathrm{T}=220 \mathrm{~K}$. This limiting undercooling is higher than the limiting undercooling of $\Delta \mathrm{T}=120 \mathrm{~K}$ for Ni dendrites [17], but is comparable to the limiting undercooling of $\Delta \mathrm{T}=190 \mathrm{~K}$ for Fe dendrites [18]. There are two reasons for the enlarged limiting undercooling for Pd dendrites than for $\mathrm{Ni}$ dendrites. The first reason is that liquid and solid $\mathrm{Pd}$ has a larger difference in Seebeck coefficient at its melting temperature (see Table 2). This larger difference is supposed to increase the transverse flow velocities and the rotational flow velocities generally allowing for sustaining of a detectable influence to a larger undercooling. A second reason is the lowering of tip velocities of Pd dendrites by the impurities dissolved in the undercooled melt. Although the TEMHD flow velocities are enlarged with rising undercooling due to enlarged thermal gradients along the dendrite/liquid interface, their influence on the tip velocities can be seen only when they are considerably larger than the tip velocities [18]. In this sense, the lowered tip velocities by the impurities are supposed to be beneficial to the observations of the TEMHD flow effects. There are more than 10 kinds of impurities in the commercial Pd material. For this large number of the species, it is rather difficult to identify the 
one that plays a dominant role in lowering the tip velocities. It is also difficult to evaluate the magnitudes of the flow velocities by fitting a dendritic growth theory with convection to the measured tip velocities [18].

More importantly did the present measurements allow us to fully verify the TEMHD flow effects that were predicted by the recent numerical simulation [15]. Here we focus our attention on the coarsening effect only because the transverse flow effect and the rotational flow effect were already observed and understood in previous studies of $\mathrm{Ni}$ and Fe dendrites $[15,17,18]$. The observation of the coarsening effect on the Pd dendrites has two reasons. First, it is benefited from the enlarged tip radii of Pd dendrites compared to those of Ni dendrites at low undercoolings. Although an insitu observation of tip radii of undercooled dendrites of metals remains challenging, it was shown elsewhere $[21,22]$ that for low undercoolings, e.g. $30 \mathrm{~K}$, tip radii of $\mathrm{Ni}$ dendrites solidifying from dilute alloys are typically by one order of magnitude smaller than those of Ni dendrites from a highly pure Ni melt (99.99\% purity). In the present experiment, the Pd dendrites were grown from the undercooled melt, which had dissolved impurities. Their tip radii are supposed to be smaller than those of $\mathrm{Ni}$ dendrites solidifying from an undercooled melt of higher purity (99.99\% purity). Such smaller tip radii may have a critical influence on the interactions of TEMHD flows with the $\mathrm{Pd}$ dendrites. If undercoolings are similar, smaller tip radii of the $\mathrm{Pd}$ dendrites meant that larger thermal gradients are established along the dendrite/liquid interface. Assuming the chemical effect of impurities on the Seebeck coefficients of solid and liquid Pd can be neglected, the larger thermal gradients will increase 
thermoelectric currents and therefore the rotational flow velocities at the tips of the $\mathrm{Pd}$ dendrites. As the magnetic field intensities are increased, the electromagnetic damping will suppress the transverse flow and confine the rotational flow into a thin liquid layer (see Fig. 1c). In this case, the viscous boundary layer falls down onto the scale of the current boundary layer. As a result, the tip velocities of the Pd dendrites are depressed again by the static magnetic fields. There may be a second reason for the observation of the coarsening effect on the tip velocities of the Pd dendrites. This second reason is linked to thermophysical properties of liquid and solid Pd. As already suggested, liquid and solid Pd has a larger difference between their Seebeck coefficients, which is by a factor of 2.7 larger than that between those of solid and liquid Ni. This larger difference can increase the rotational flow velocities around the tips of the Pd dendrites in terms of a model presented elsewhere [18]. On the other hand, liquid Pd has a smaller dynamic viscosity than that of liquid Ni at respective melting temperatures while it has an electrical conductivity comparable to that of liquid Ni. Such thermophysical properties of liquid Pd can cause stronger TEMHD flows, especially the rotational flows, at moderate magnetic field intensities. For these two reasons, the rotational flows are supposed to be stronger for Pd dendrites to such an extent that they can be damped at a lower magnetic field intensity than that predicted for Ni dendrites. As a result, the coarsening effect of the confined rotational flows on the Pd dendrites becomes detectable at the highest magnetic field intensity of $\mathrm{B}=6 \mathrm{~T}$ applied in the experiment.

The possible shift of the coarsening effect on the tip velocities of the Pd dendrites 
to higher magnetic field intensities for intermediate undercoolings can be interpreted by considering the opposite argument. As undercooling increases to $\Delta \mathrm{T}=70 \mathrm{~K}$ or above, the tip velocities of the Pd dendrites rise up due to solute trapping. This rapid increase is already significant before a complete trapping of impurities occurs at the $\Delta \mathrm{T}_{\text {crit }}=110 \mathrm{~K}$. Then, the tip kinetics of the Pd dendrites is supposed to be more similar to that of thermal dendrites than that of a dilute alloy dendrite [21, 22]. A direct consequence of this change of the growth kinetics is the recovery of large tip radii of the $\mathrm{Pd}$ dendrites. Then, the thermal gradients along the dendrite/liquid interface become smaller than those at low undercoolings. In this case, the rotational flow velocities are smaller so that the coarsening effect on the tip radii is shifted to higher magnetic field intensities, i.e. $\mathrm{B}>6 \mathrm{~T}$.

\section{Conclusions}

In summary, dendrite tip velocities in the undercooled melt of a commercially pure Pd material have been measured under static magnetic fields of intensities up to $B=6$ T. A two-staged undercooling dependence of the tip velocities observed for each magnetic field intensity has been attributed to unknown impurities dissolved in the undercooled Pd melt. The variations of the tip velocities with rising magnetic field intensity have provided complete evidence for the three kinds of TEMHD flow effects on dendritic growth predicted numerically [15]. While the first depression followed by the recovery of the tip velocities with rising magnetic field intensity verifies the significant effects of a transverse flow around the tip and then a rotational flow 
around its axis, the second depression of the tip velocities at low undercoolings at the highest magnetic field intensity of $\mathrm{B}=6 \mathrm{~T}$ has verified for the first time the coarsening effect, which was also predicted by the numerical simulations [15]. The observations of this coarsening effect has been attributed to a large difference between Seebeck coefficients of solid and liquid Pd as well as the impurities dissolved in the undercooled liquid.

\section{Acknowledgement}

This work is financially supported by the National Natural Science Foundation of China (51071043 and 51211130113). The authors thank Jiayun Cai, Jia Liu, Xuewei Zhao, Mengkun Han, Weina Zhao and Dandan Zhao for their assistance in the experimental work. The authors also thank Jan Gegner and Sven Binder for providing access to the computer codes for video image analysis. 


\section{References}

[1] J. A. Shercliff, Thermoelectric magnetohydrodynamics, J. Fluid Mech. 91 (1979) $231-251$.

[2] J. A. Shercliff, Thermoelectric MHD with walls parallel to the magnetic field, Int. J. Heat Mass Transf. 23 (1980) 1219-1228.

[3] R. P. Silberstein, D. J. Larson Jr, B. Dressler, Thermoelectric and morphological effects of Peltier pulsing on directional solidification of eutectic Bi-Mn, Metall. Trans. A 15 (1984) 2147-2154.

[4] L. L. Zheng, D. J. Larson Jr, Thermoelectric effects on interface demarcation and directional solidification of bismuth, J. Cryst. Growth 180 (1997) 293-304.

[5] S. Battaglioli, A. J. Robinson, S. McFadden, Influence of natural and forced gravity conditions during directional columnar solidification, Int. J. Heat Mass Transf. 126 (2018) 66-80.

[6] T. Alboussiere, R. Moreau, D. Compte, Influence of a magnetic-field on the solidification of metallic alloys, Comptes Rendus de Lacademie des Sciences Serie II, 313 (1991) 749-755.

[7] Y. Y. Khine, J. S. Walker, F. R. Szofran, Thermoelectric magnetohydrodynamic flow during crystal growth with a moderate or weak magnetic field, J. Cryst. Growth 212 (2000) 584-596.

[8] P. Lehmann, R. Moreau, D. Camel, R. Bolcato, Modification of interdendritic convection in directional solidification by a uniform magnetic field, Acta Mater. 46 (1998) 4067-4079.

[9] X. Li, Y. Fautrelle, Z. Ren, Influence of thermoelectric effects on the solid-liquid interface shape and cellular morphology in the mushy zone during the directional solidification of Al-Cu alloys under a magnetic field, Acta Mater. 55 (2007) 
$3803-3813$.

[10] I. Kaldre, Y. Fautrelle, J. Etay, A. Bojarevics, L. Buligins, Thermoelectric current and magnetic field interaction influence on the structure of directionally solidified Sn-10wt\%Pb alloy, J. Alloys Compd. 571 (2013) 50-55.

[11] J. Wang, Y. Fautrelle, H. Nguyen-Thi, G. Reinhart, H. L. Liao, X. Li, Y. B. Zhong, Z. M. Ren, Thermoelectric magnetohydrodynamic flows and their induced change of solid-liquid interface shape in static magnetic field-assisted directional solidification, Metall. Mater. Trans. 47 (2016) 1169-1179.

[12] A. Kao, B. Cai, P. D. Lee, K. Pericleous, The effects of thermoelectric magnetohydrodynamics in directional solidification under a transverse magnetic field, J. Cryst. Growth 457 (2017) 270-274.

[13] A. Kao, Thermoelectric magnetohydrodynamics in dendritic solidification. University of Greenwich, United Kingdom, 2010.

[14] A. Kao, G. Djambazov, K. Pericleous, V. Voller, Thermoelectric MHD in dendritic solidification, Magnetohydrodynamics 45 (2009) 267-277.

[15] A. Kao, J. Gao, K. Pericleous, Thermoelectric magnetohydrodynamic effects on the crystal growth rate of undercooled Ni dendrites, Philos. Trans. R. Soc. A, 376 (2018) 20170206.

[16] J. C. Chen, Y. H. Wei, X. H. Zhan, C. Gu, X. Y. Zhao, Thermoelectric currents and thermoelectric-magnetic effects in full-penetration laser beam welding of aluminium alloy with magnetic field support, Int. J. Heat Mass Transf. 127 (2018) $332-334$.

[17] J. Gao, M. K. Han, A. Kao, K. Pericleous, D. V. Alexandrov, P. K. Galenko, Dendritic tip velocities in an undercooled melt of pure nickel under static magnetic fields: A test of theory with convection, Acta Mater. 103 (2016) 184-191. 
[18] R. J. Zhao, J. Gao, A. Kao, K. Pericleous, Measurements and modelling of dendritic tip velocities of pure Fe with thermoelectric magnetohydrodynamics convection, J. Cryst. Growth, 475 (2017) 354-361.

[19] J. Gao, A. Kao, V. Bojarevics, K. Pericleous, P. K. Galenko, D. V. Alexandrov, Modeling of convection, temperature distribution and dendritic growth in glassfluxed nickel melts, J. Cryst. Growth 471 (2017) 66-72.

[20] K. Eckler, R. F. Cochrane, D. M. Herlach, B. Feuerbacher, M. Jurisch, Evidence for a transition from diffusion-controlled to thermally controlled solidification in metallic alloys, Phys. Rev. B 45 (1992) 5019-5022.

[21] P. K. Galenko, S. Reutzel, D. M. Herlach, D. Danilov, B. Nestler, Modelling of dendritic solidification in undercooled dilute Ni-Zr melts, Acta Mater. 55 (2007) $6834-6842$.

[22] S. Reutzel, Magnetische Messungen an unterkühlten Co-Basis Schmelzen, PhD Thesis Ruhr-University Bochum, 2002, 1-140.

[23] T. Iida, I. L. Guthrie, The Physical Properties of Liquid Metals, Oxford Univ. Press, New York, 1988.

[24] T. Iida, R. Guthrie, M. Isac, N. Tripathi, Accurate predictions for the viscosities of several liquid transition metals, plus barium and strontium, Metall. Mater. Trans. B 37 (2006) 403-412.

[25] B. C. Dupree, J. B. van Zytveld, J. E. Enderby, The resistivity and thermoelectric power of solid and liquid palladium, J. Phys. F: Met. Phys. 5 (1975) L200-L202.

[26] J. E. Enderby, B. J. Dupree, The thermoelectric power of liquid Fe, Co and Ni. Philos. Magn. 35 (1977) 791-793. 


\section{List of Figure Captions}

Fig. 1 Schematic illustration of three kinds of TEMHD flows close to the primary tip of an undercooled dendrite in sequence of rising magnetic field intensity, B. Details of the flow patterns can be found in Ref. 15 .

Fig. 2 Experimental set-up for measurements of tip velocities of undercooled dendrites under static magnetic fields. (a) Side view (b) schematic configuration.

Fig. 3 Schematic illustration of the contour of an undercooled dendrite and a thermal front inside a spherical sample. Red zone: a semi-solid zone comprising a skeleton of the undercooled dendrite and interdendritic liquid; orange zone: the thermal diffusion zone; blue zone: the undercooled liquid. A yellow arrow shows a thermal front traveling distance, $S_{i}$, at traveling time, $t_{i}$.

Fig. 4 Illustration of high-speed video images of the top surface of the Pd sample under different conditions of bulk undercoolings $(\Delta \mathrm{T})$ and magnetic field intensities (B). In each image, a red circle marks the sample surface, a yellow circle represents a simulated thermal front, and a yellow dot shows the location of a crystal nucleus on the bottom surface of the sample. A zone with bright contrast corresponds to the recalescing part, a zone with dark contrast corresponds to the undercooled liquid, and a boundary layer between them is not resolved due to a small width. Traveling 
distances of the thermal front at different time are plotted in a panel on the right side of each row of images.

Fig. 5 Measured tip velocities of Pd dendrites under magnetic field intensities of $\mathrm{B}=$ 0-6 T. Solid lines represent power law fittings to the measured data at low $(<110 \mathrm{~K})$ and high $(>110 \mathrm{~K})$ undercoolings. Coefficients of the fitted power laws are listed in Table 1.

Fig. 6 Magnetic field dependences of normalized tip velocities of Pd dendrites at various undercoolings. (a) $\Delta \mathrm{T}=30-110 \mathrm{~K}$ (b) $\Delta \mathrm{T}=120-220 \mathrm{~K}$. 
(a)

Low magnetic field

(transverse flow)

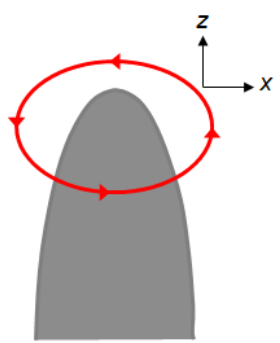

Hot fluid flows onto a tip oriented along the $z$ axis. The transverse component of a tilted magnetic field is parallel to the $x$ axis. (b)

Moderate magnetic field (rotational flow)

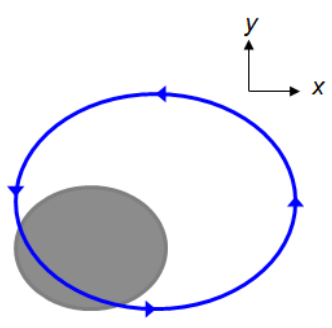

The transverse flow combines itself with a rotational flow about the $z$ axis due to the parallel component of the tilted field. The latter flow introduces cold fluid onto the tip. (c)

High magnetic field (confined flow)

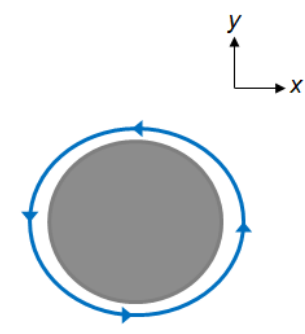

The transverse flow is damped, leaving the rotational flow confined to the tip and causing tip coarsening.

Fig. 1

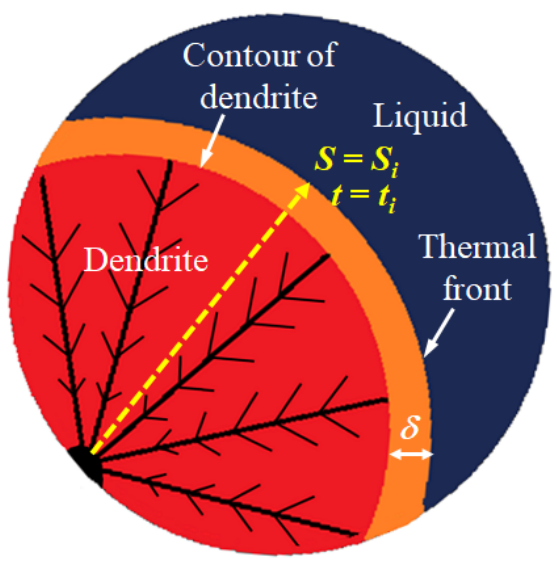

Boundary laver:

$\delta \leq 100 \mu \mathrm{m}$

Tip velocity:

$$
V=\frac{\Delta S}{\Delta t}
$$

Fig. 3 


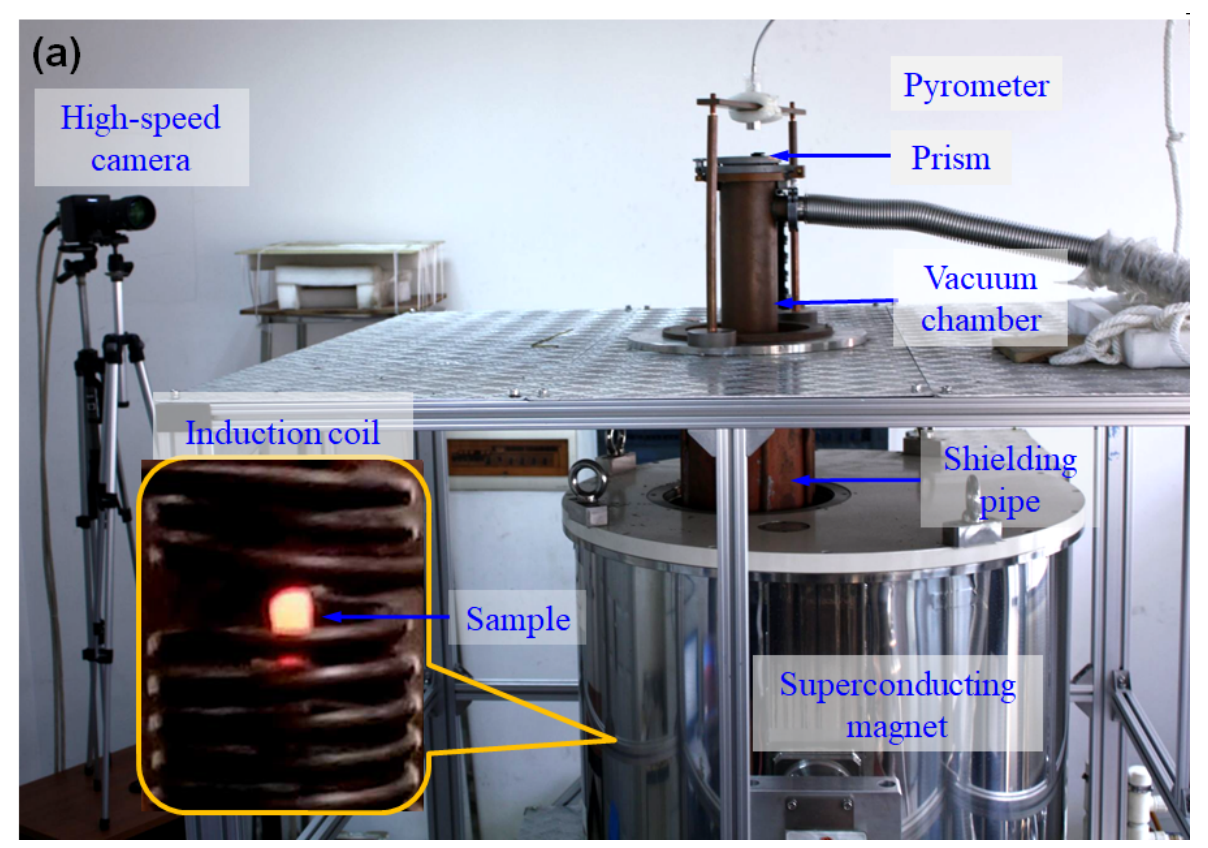

Fig. 2a

(b)

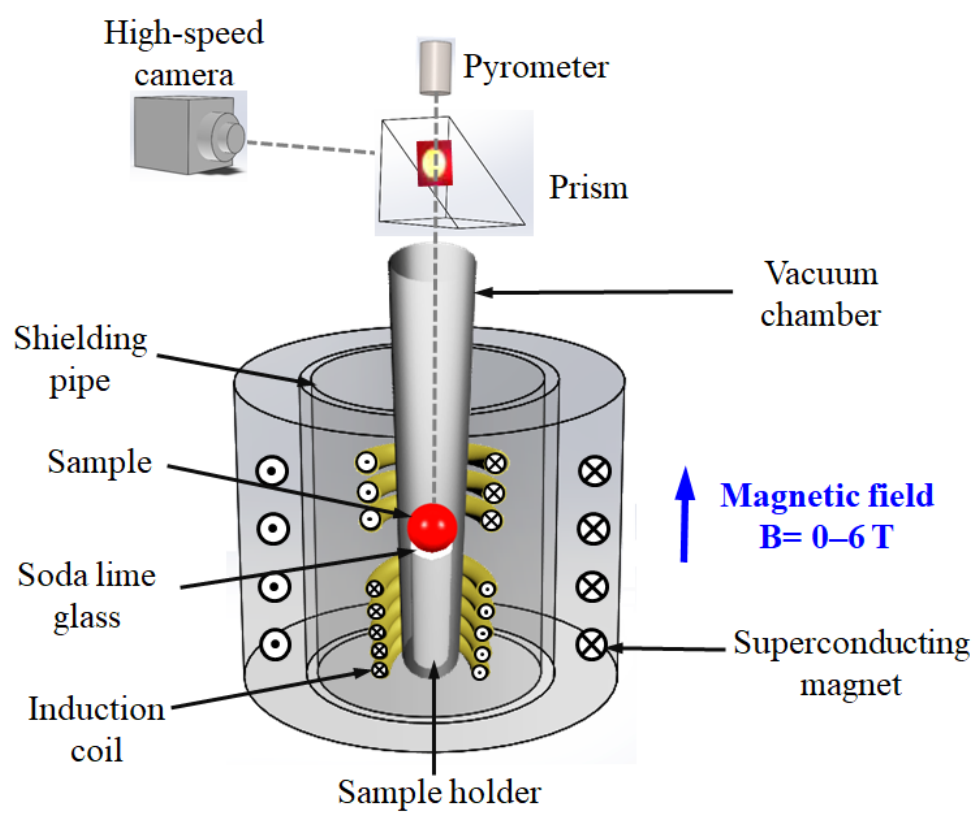

Fig. 2b 

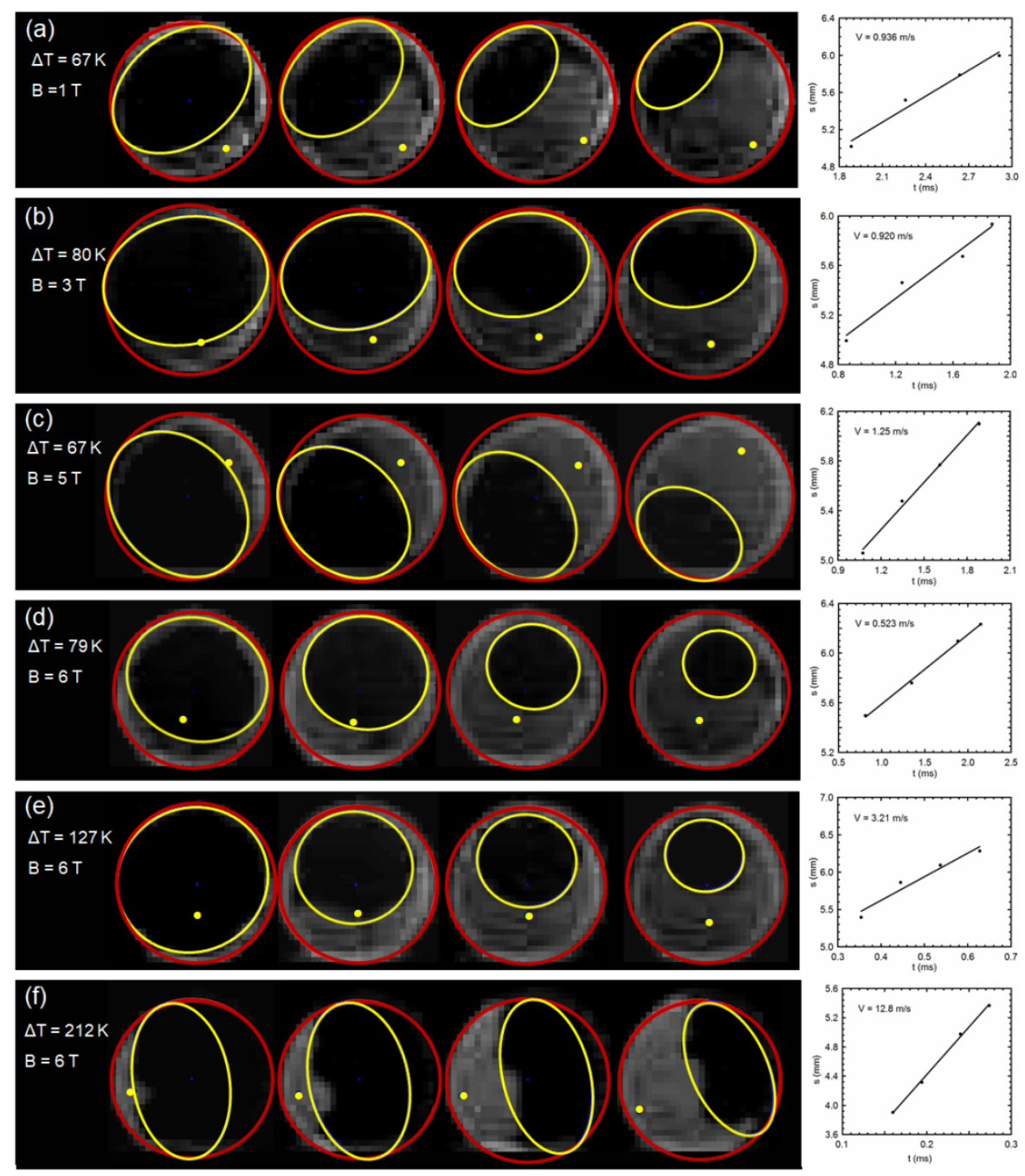

Fig. 4 


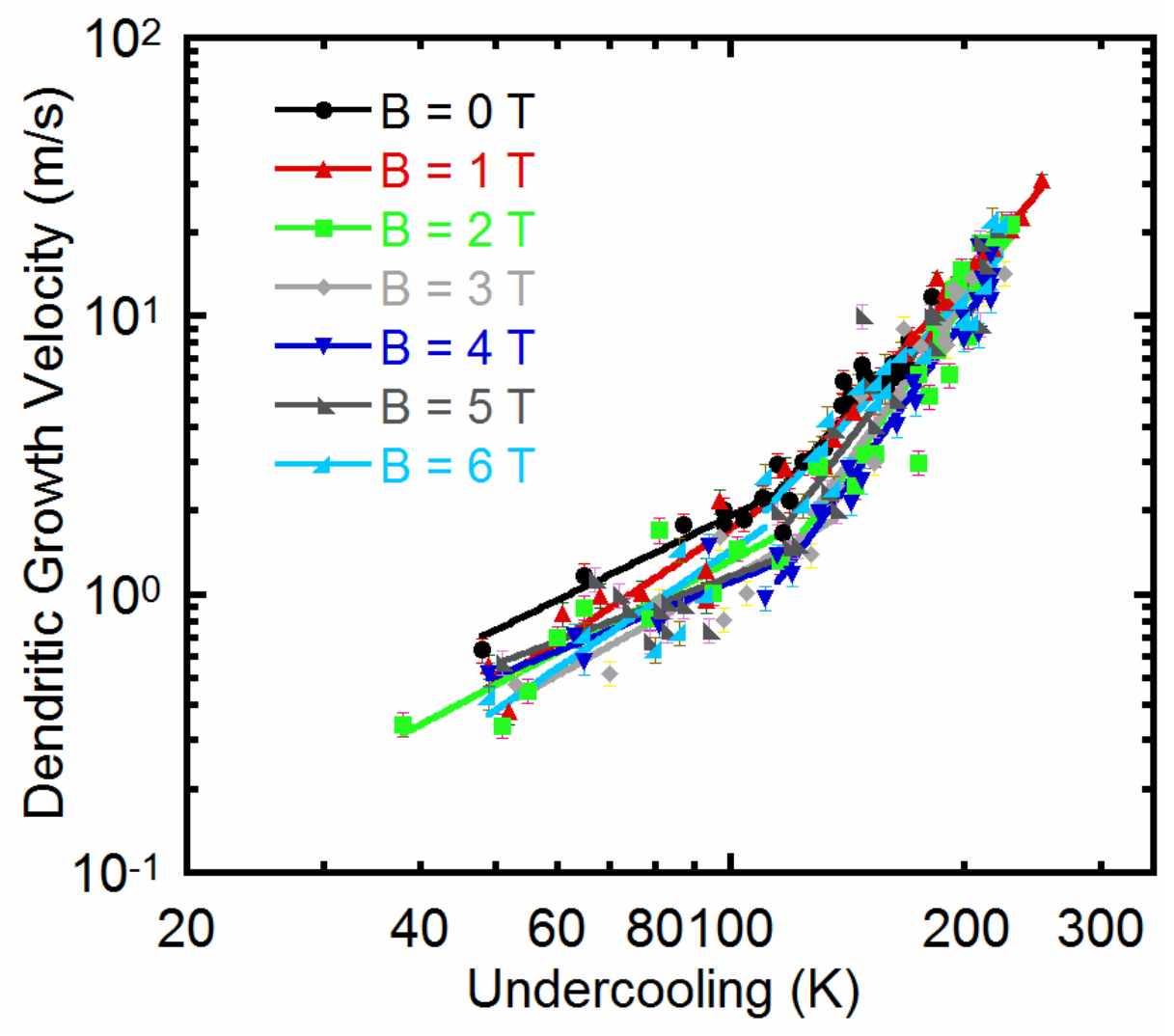

Fig. 5 


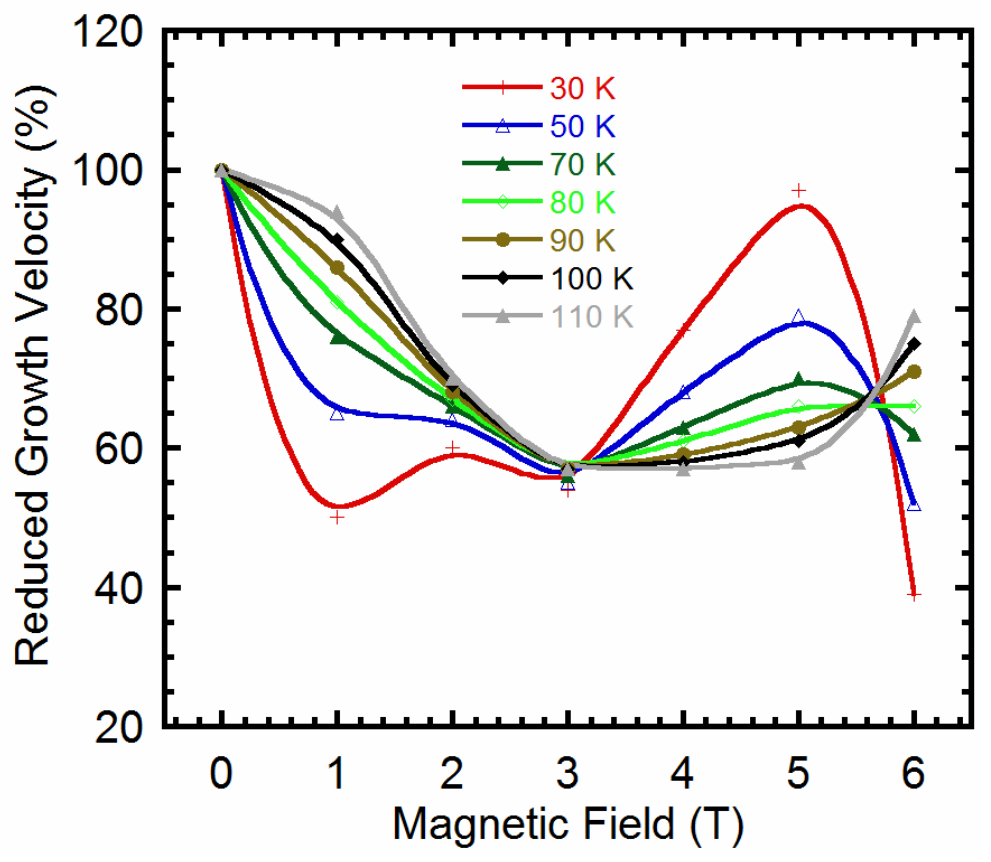

Fig. 6a

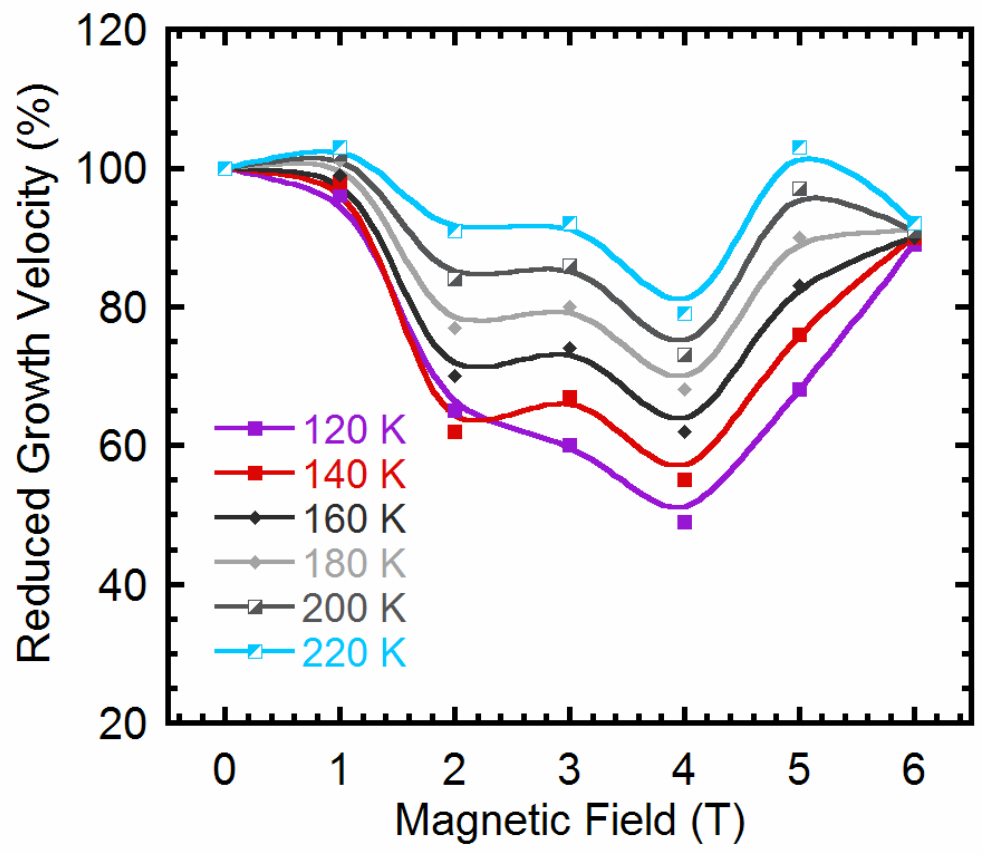

Fig. 6b 
Table 1 Parameters of power laws $V=a \cdot(\Delta T)^{b}$ fitted to the measured tip velocities of

Pd dendrites under each magnetic field for low and high undercoolings.

\begin{tabular}{|c|c|c|c|c|c|}
\hline \multirow{2}{*}{$\begin{array}{l}\text { Magnetic } \\
\text { Field } \\
\text { Intensity } \\
\text { B (T) }\end{array}$} & \multicolumn{2}{|c|}{$\begin{array}{c}\text { Power law for } \\
\Delta T \leq 110 \mathrm{~K}\end{array}$} & \multicolumn{2}{|c|}{$\begin{array}{c}\text { Power law for } \\
\Delta T>110 \mathrm{~K}\end{array}$} & \multirow{2}{*}{$\begin{array}{c}\text { Root- } \\
\text { mean- } \\
\text { square } \\
\text { deviation } \\
(\%)\end{array}$} \\
\hline & $\mathrm{a}$ & $\mathrm{b}$ & $\mathrm{a}$ & $\mathrm{b}$ & \\
\hline 0 & $3.698 \times 10^{-3}$ & 1.356 & $7.324 \times 10^{-7}$ & 3.1543 & 19 \\
\hline 1 & $3.622 \times 10^{-4}$ & 1.838 & $3.850 \times 10^{-7}$ & 3.280 & 14 \\
\hline 2 & $1.445 \times 10^{-3}$ & 1.480 & $7.450 \times 10^{-9}$ & 3.987 & 24 \\
\hline 3 & $1.759 \times 10^{-3}$ & 1.395 & $1.553 \times 10^{-8}$ & 3.854 & 23 \\
\hline 4 & $6.447 \times 10^{-3}$ & 1.117 & $8.463 \times 10^{-9}$ & 3.938 & 18 \\
\hline 5 & $1.333 \times 10^{-2}$ & 0.969 & $1.787 \times 10^{-8}$ & 3.849 & 22 \\
\hline 6 & $2.299 \times 10^{-4}$ & 1.896 & $5.121 \times 10^{-7}$ & 3.204 & 21 \\
\hline
\end{tabular}

Table 2 List of thermophysical properties of solid and liquid Pd in comparison to those of $\mathrm{Ni}$ and Fe taken from the literature [23-26].

\begin{tabular}{cccc}
\hline Parameter & $\mathrm{Pd}$ & $\mathrm{Ni}$ & $\mathrm{Fe}$ \\
\hline Difference between Seebeck & & & \\
coefficients of liquid and & $-16 \pm 5$ & $6 \pm 3$ & $-3 \pm 2$ \\
solid metal $\left(\times 10^{-6} \mathrm{~V} \cdot \mathrm{K}^{-1}\right)$ & & & \\
Electrical conductivity $\left(\times 10^{5} \Omega^{-1} \cdot \mathrm{m}^{-1}\right)$ & 12.0 & 11.8 & 7.22 \\
Dynamic viscosity $\left(\times 10^{-3} \mathrm{~Pa} \cdot \mathrm{s}\right)$ & 4.22 & 5.64 & 6.37 \\
\hline
\end{tabular}




\section{Conflict of Interests}

All authors, Mr. Rijie Zhao, Dr. Jianrong Gao, Dr. Andrew Kao and Dr. Koulis

Pericleous, state that there is no conflict of interest. 